\title{
Voice Controlled Personal Assistive Bot with Object Detection
}

\author{
${ }^{1}$ S. A. Sivasankari, ${ }^{2}$ K. V. B. N. S. Chandra Mouli, ${ }^{3}$ B. Rajyalakshmi, ${ }^{4}$ B. Goutham Kumar \\ Reddy \\ ${ }^{1}$ Associate Professor, ${ }^{2,3,4} \mathrm{UG}$ Student \\ Department of ECE, Koneru Lakshmaiah Education Foundation, Green Fields, Vaddeswaram, \\ Andhra Pradesh 522502, India
}

Article History: Received:11 January 2021; Accepted: 27 February 2021; Published online: 5 April 2021

\begin{abstract}
Currently innovation has made our lives simpler for individuals. Be that as it may, from this innovation, certain gatherings of individuals need more assistance and backing for old or handicap individuals. This innovation can make a method of having a typical life. Thus, we zeroed in on the idea of an individual colleaguerobot.Thefundamentalobjectistosupplyhelptodebilitatedpeople. ThisPersonalassistiveBot help to decrease the manual endeavors being put by people in their everyday errands. The intention is to execute a specialized work that is voice controlling one which can act as a PAthat can performvarious errands or administrationsforaperson.Thisisuncommonlyintendedforthisgroupofpeopleasitsprimaryreasonfor existing is to supply help to relate senior or debilitated individual. The human voice order is given to themechanicalrighthanddistantly, byutilizingavoiceorder. Theautomatonwillperformdifferentmovements:

Forward, Backward, Right, Left and start/stop activities. The robot can likewise peruse and perceive the letter sets and text and the words which are said by the person will check from the google dictionary and printasatext.Thecapabilityoftherobotistodetecttheobjectsandrelocatethemfromoneplacetoanother and includes the face recognition. So, our main ideology is to create a personal assistance bot, which is capable of handling small objects. We are planning to make the bot consisting of four wheels and an arm placed on top. Using Raspberry $\mathrm{Pi}$, we are communicating the sensors and motors throughour voice commands.SmartassistantslikeGoogleforandroid,SiriforApple,CortonaforMicrosoft, theseassistive givesusaplatformtocommunicatetoabot.AsweareprogrammingonPython,AmodulenamePyaudio will helptocommunicatewithabotandhavingtheextrafeaturelike'SpeechtoText'. Andwewouldlike to add an extra feature like object and person detection. A Camera module will be installed for capturing videoandrecognizetheHumansandobjectscarriedoutwithMachineLearningAlgorithm
\end{abstract}

\section{Keywords: Face Recognition, Voice Recognition, Object Detection}

\section{INTRODUCTION}

Weareplanningtomakethebotconsistingoffourwheelsandanarmplacedontop.UsingRaspberry Pi,weare communicatingthesensorsandmotorsthroughourvoicecommands.SmartassistantslikeGoogleforandroid, Siri for Apple, Cortona for Microsoft, these assistive gives us a platform to communicate to a bot. As we are programming on Python, A module name Pyaudio will help to communicate with a bot and having the extra feature like 'Text to Speech' and 'Speech to Text'. And we would like to count an extra feature like object and persondetection.ACameramodulewillbeinstalledforcapturingvideoandrecognizetheHumansandobjects carried out with Machine Learning Algorithm. The Components that we are used to construct a robot are Raspberry Pi 3 - Model B+ - 1.4Ghz 64GhzProcessor with 1 GB Ram [16], wheels, Motors-200/300 rpm, motor driver-L293D. We will use robotic arm to hold an object and the rotation of arm will be controlled by the servo motors. Generally, Robots are used nowadays to perform all the undertakings with better productivity. This makes the growth both in Industrial level and home level. This Personal assistive bot will be helpful in our daily life Individual robot assists with diminishingthemanualeffectsofpeopleintheireverydayundertakings. Theassistive

robot which obeyed the voice requests and act based on the requests. The voice communication with robots is achieved through blue tooth devices[2].Therobots which are designed here is four wheeled Robots which includesacameraandrobotized arm.ThecameraisusedforFaceRecognition, area, object, space anddistanceassessment, andamechanicalarmtoperform pickandspotexercises.Itwillingeneralbeforthemostpartusedinvariousapplications, forexample,substance ventures,medicalcareforthedebilitatedandoldpopulace. 


\subsection{Voice Recognition}

ThisVoiceRecognitionmakesamajorroleinthisprojectwiththehelpofvoicecommandsthebotwillmoveor takeanobject,VoicecommandswillgivethroughmicrophoneheretheBluetoothactsasamicrophoneaswell asaspeaker[7]. Withthehelpofpyaudiomodulewewillgenerateacodeonpythonandcommunicatewithabot. It will take our audio as an input via microphone and the output as a text. It is very useful area having many applications in our daily life. Discourse acknowledgment empowers a PC to catch the words verbally expressed

byahumanwiththehelpofmicrophone [3].Whenwegiveourvoicecommandstothebotwiththehelpofspeech recognition module in the pyaudio and we need to install setup tools-wheels in the command prompt so that it won'tget anyerrorswhilewerunthecodeaftercompletionofallprocesswewillgivevoicecommands[14] and voice commands which we given will search from google dictionary and the words will be print as a text, and with the voice commands which was given by us to the bot with that voice commands there is a noise w.r.t the voicethatnoisewillberemovedbytheambientnoisethiswillhelptoremovetheunnecessarynoise[7].

\subsection{FaceRecognition}

Facialrecognitionisthebestnewtechnologiesintheworld.Thetechnologythatismosteffectiveisrecognizing anindividual.Humanshavedifferentwaystoidentifyaperson.Astechnologyisdeveloping withthepresentera tomakeitmoreconvenient.InthisprojectthefacialrecognitionprocessdonewithMachinelearningalgorithm. firstitwilltakepicturesofapeoplewiththehelpofcamera.Itwilltakevideofeedofpeoplesaveasafolder thatfolderthepicturesthataretakesasavideofeedwillbestoreditiscapabletotakenearly 100picturesand canstore.TofindthepictureofthepersonatfolderitwilltakeGracescalepicturesorvalueanditwillsaveon memory card and then this all will train by the LBPH Algorithm where LBPH-Local Binary Pattern Histograms.LBPHalgonithm must be used forhuman facerecognition in real time. This algoithm which has characteristics like low level resolution etc.[9].

\subsection{ObjectDetection}

At the end, the cons of the existing object detection models are included. The existing models are unfit for smart cities and the current data sets. At the same it can't cover all classes of smart cities with best solution[1].Theframework

utilizesultrasonicsensorsforlimitdetectingandworksasneedsbe, ObjectidentificationisaPCvisionmethod in which a product framework can identify and follow the item from a given picture or video or objects around

surrounding.Theuniquepropertyaboutitemidentificationisthatitrecognizestheclassofarticleandtheirarea explicitdirections[15].Theareaisbroughtupbydrawingaboxaroundtheobject.Thecapacitytofindtheitem insideapicturecharacterizestheexhibitionofthecalculationutilizedforidentification.Faceidentificationisone oftheinstancesofobjectrecognitionaftertheobjectrecognitiontheobjectwillbedetectedthroughtheultrasonic sensor.Thiswillhelptodetecttheobjects.

\subsection{RoboticArm}

The Robotic arm is designed by of seven metal segments which are joined by six joints, the computer controls the robot. These robots use motion sensors. This type of robot has six degrees of freedom, the arms job is to move hand from one place to another place. Most industrial robots work with auto assembly lines. It takes the incredibly precise hand in a tiny microchip. The industrial robotic arm, it is made of steel or cast iron. In this stepmotorisusedanditwasconnectedtoeachjoint,roboticarmsareusedtoliftbigitemthereare7kindsof automated arms, Cartesianrobot, Cylindrical robot,Spherical robot, alarm robot, Articulated robot, Parallel robot, Anthropomorphic robot. The voice inputs are given in a microphone to the voice recognition [13]. The output is in voice command is given to the microcontroller will generate the control signals to operate the four motorsoftheroboticarm, thestructureandtheycanfluctuatefromvariousplans. Thiswillallowthecomputer tomovethearm,repeatingexactthesamemovementrepeatedly. Therobotusemotionsensors. TheAndroidphone and robotic arm are with Bluetooth module. The hand movements are used with servo motors which is at specific rotations and positions. The three modules of robot are as follows: speech command recognition module, object classifier module and robotic gripper arm module, It is very easy to control the robot by using voice commands. Accurately can control the robot by Voices. First Robot grabs the object by taking the voice command and find the object using the object classifier module. And at the end, it holds and replaces the object using the robotic gripper arm module. 


\section{LITERATUREREVIEW}

Ayesha Shafiq, Humera Tariq, FareedAlvi and Usman Amjad (2019) et.al introduced voice recognition model. They designed the model by using MEL frequency Cepstrum coefficient. To recognize the voice, they followed Hidden Markov model.

PiyushVashistha(2019) had introduced the voice controlled personal assistive bot with different methods those are voice control, character control, virtual control. The Voice controlled PA is acquired by the Raspberry Pi board.Raspberry Pi board is used here for Voice controlling personal assistant.PA can be controlled intelligently and artificially by the directed pre voices. We need to install OS in it so that it will communicate with sensors.

AftabAhmed,JiandongGuo,FayazAli(2018)haddesignedtheFacialrecognitionisthebestnewtechnologies intheworld.Thetechnologythatismosteffectiveisrecognizinganindividual.Humanshavedifferentwaysto identifyaperson.Astechnologyisdevelopingwiththepresenteratomakeitmoreconvenient.Inthisprojectthe facialrecognitionprocessdonewithMachinelearningalgorithm.

JishnuUK,InduV,KJAnandakrishnan (2019)had introducedthepersonalassistivebotisconstrainedbyvoice orders to pick articles and Voice correspondence fromthe robot toandroid cell phone and vice versais done through Microphone.Theproposedfourwheeledrobotcomprisesof acameraandmechanicalarm.

Anurag Mishra, (2020) has introduced the controlling of voice commands and communication with robot using Bluetooth and it acts as a microcontroller and with the help of control logic the robic arm control, robotic body control, robotic hand control will be done. This paper portrays the plan and advancement of an individual colleague robot, which is constrained by voice orders to pick long/short distance objects. Voice transmission activity can be carried out by use of microphone between Robot and devices. The proposed four wheeled robot comprises of a camera and automated arm.

\section{HARDWAREDESIGN}

O RaspberryPi3-ModelB+- 1.4Ghz64GhzProcessorwith1GBRam

O Wheels-4

O Motors-4-200/300rpm

O MotorDriver(L293D)

O ServoMotor-4

O ConnectingWires

O WebCamera

O Microphone

\section{Raspberry Pi}

This Raspberry Pi mainly includes the below features and it has GPIO (general purpose input/output)pinswhich controls the electronic component for computing physically and for IoTIthassingleUSBconnector; ithas40pins. With the help of 1 GB SDRAM the raspberry pi model was designed.

\section{Motor Driver}

The L293D is a 16-pin Motor Driver IC is very simple. Single L293D IC can run two DC Motors at the same time. Left input pins will regulate across left side and right input pins will regulate across right-hand side. 


\section{BLOCKDIAGRAM}

Fig.4.1 BLOCK DIAGRAM



\section{WORKINGPRINCIPLE}

Afacialacknowledgmentframeworkwhich uses the biometrics and the face identification and the characteristics are highlighted from the photos or videos. This framework contrasts the available data and discover the match from all the basic information's and are realized in the end. Basically, the facial acknowledgement which involves in the business applications too. This business applications provides everything from ground observation till marketing advertising.Fundamental rule of voice acknowledgment includes the way that discourse or words expressed by any individual reason vibrations in air, known as sound waves. These constant or simple waves are digitalized and handled and afterward decoded to fitting words and afterward proper sentences.

when coming to the second part of the project Face Recognition. The first step is to register the face of the person whose face should be detected and proceed to the next process. we will be adding a data set the (set of photos of that person) that will store in a folder and the system will train itself for the proper detection of theface.wheneverthesystemisonandifitfindsafaceitwillcheckforthefacethatisregisteredanditgivesvalue thatiscalledasconfidence. Theconfidencelevelbasedonthesimilarityoftheedgesofthefacebasingonthat if that is similar the value will be less than 100 and else it will be greater than 100.If the value of confidence is lessthan 100percentthanthefacethatisdetectedisthefacethatshouldberecognised,anditdisplaysthename of the person that we have registered previously. If the value of the confidence is greater than 100 then the face is different, and it is ignored by the Robot. And the future scope of the project is to recognisean object that we want and process the work that we have given and the other is to recognisean object and to describe and show the information of thatobject. 


\section{PROPOSEDARCHITECTURE}

Fig. 6.1 FACE RECOGNITION

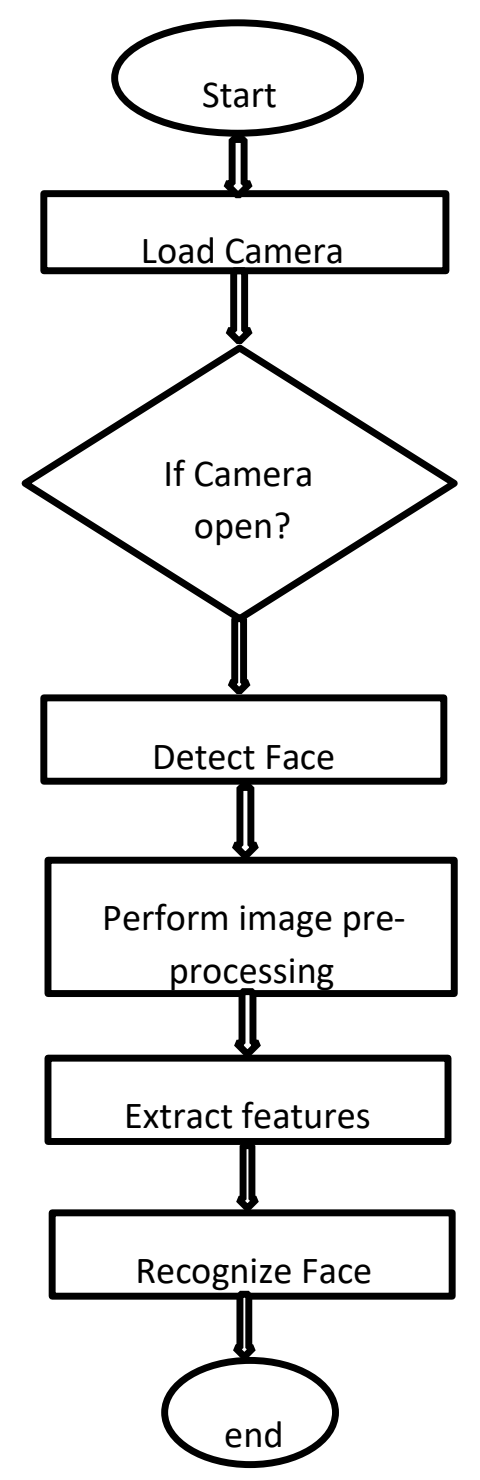

Fig. 6.2 VOICE RECOGNITION

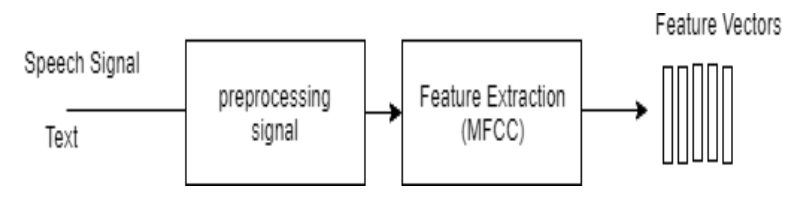




\section{SIMULATIONRESULTS}

Fig. 7.1 VOICE RECOGNITION

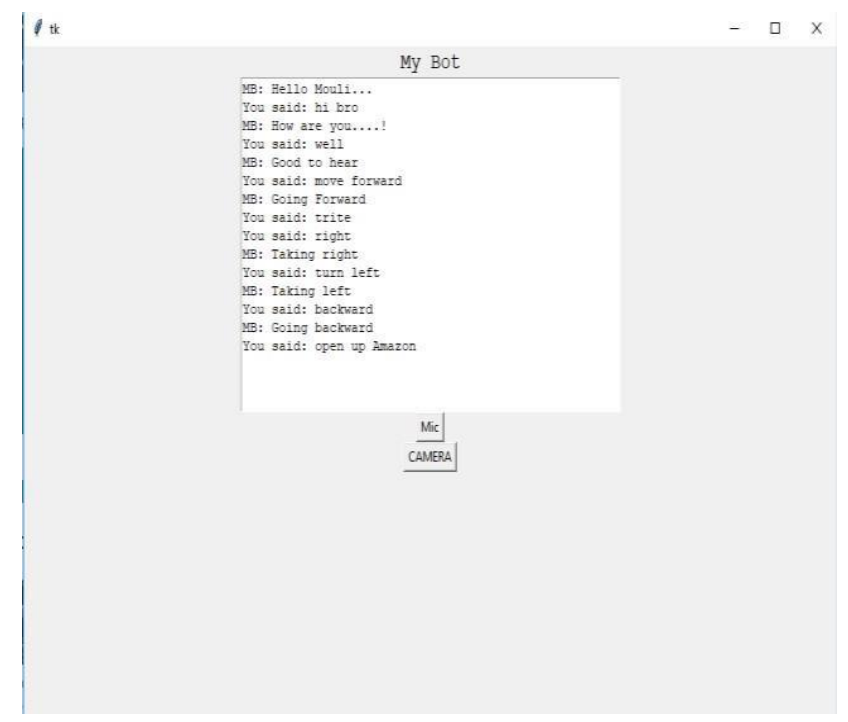

Fig. 7.2 FACE RECOGNITION

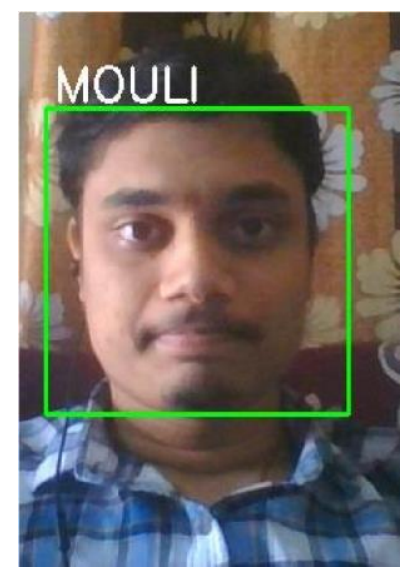

\section{ACKNOWLEDMENTS}

We are very thankful to DR. SUMANMALOJI, Head of the Department for Electronics \& Communication Engineering who provides better space tocomplete our projectwork and our journal work. We would like to thank to the beloved honorable President \&Vice Chancellor, KLUniversityforprovidingthenecessaryfacilitiestocarrytheconcludedworkofours.

Last but not least, weare sincerely thanking to Dr.S.A. SIVASANKARI (Associate professor) for her valuable time, guidance and help till the completion of our work. Without here guidance and cooperation our work could not be completed well to taste the results.

\section{REFERENCES}

1. Asra Aslam, Edward Curry image and vision computing L. R. Rabiner, B. H. Juang, "Fundamentals of Speech Recognition", Prentice Hall, Englewood Cliffs, New Jersey,1993.

2. L.Diener,S.BredehoeftandT.Schultz,"AcomparisonofEMG-to-SpeechConversionfor

3. Isolated and Continuous Speech," Speech Communication; 13th ITG-Symposium, Oldenburg, Germany, 2018, pp. 1-5.

4. PiyushVashisthaJuginderPalSinghGLAUniversity-RaspberryPibasedvoice-operatedpersonalassistant TextBook:IntroductiontoRobotics:MechanicsandControl,byJohnCraig

5. TEXT BOOK :Understanding Machine Learning: From Theory to Algorithms, by y Shai Shalev- 
Schwartz and Shai Ben-David

6. PRAKASH, P., \& AR, A.A REVIEW ON DIFFERENT APPROACHES TO EFFICIENTLY DETECT AND RECOGNIZE INFORMATION FROM TRAFFIC PANELS.

7. Peter X. Liu, A. D. C. Chan, R. Chen, K. Wang, Y. Zhu Department of Systems \&Computer Engineering CarletonUniversityOttawa,ONCanada,K1S5B6

8. NaradaWarakagoda "Hidden Markov Models" http://jedlik.phy.bme.hu/ gerjanos/HMM/node2.htm 1, May 10,1996

9. Nazarieh, M. (2016). A brief history of metacognition and principles of metacognitive instruction in learning. BEST: Journal of Humanities, Arts, Medicine and Sciences, 2(2), 61-64.

10. AftabAhmedSchoolofInformation\&SoftwareEngineering,UniversityofElectronicScience\&Technolog y of China Chengdu,China

11. J.OlshausanBA,FieldDJ.Emergenceofsimple-cellreceptivefieldpropertiesbylearningasparsecodefor naturalimages.Nature,1996,381(6583):607-609

12. JishnuUKDeptofElectricalandElectronicsEngineering,AmritaSchoolofEngineeringAmritapuri,India

13. Ass.Prof.EmadS.Othman,SeniorMemberIEEE - Region 8, "Voice Controlled Personal Assistant Using Raspberry Pi”, International Journal of Scientific \& Engineering Research Volume 8, Issue 11, November2017 1611, ISSN 22295518,pp1611 -1615.

14. TALREJA, S. (2016). Stochastically optimized handwritten character recognition system using Hidden Markov Model.

15. Chien-Wei Chen, Rui-Ming Hong, Hung-Yu Wang Department of Electronic Engineering National KaohsiungUniversityofAppliedSciencesKaohsiung807,Taiwan,R.O.C.

16. HUSSEIN, M. J. K. A. I. RECOGNIZING THE IRAQI LICENSE PLATE USING STATISTICAL.

17. AnuragMishra, PoojaMakula, AkshayKumar, KritKaran and V. K. Mittal Indian Institute of Information Technology, Chittoor,SriCity, A.P., India

18. Hamdan, S. A. L. A. M., \&Shaout, A. D. N. A. N. (2016). Face Recognition Using Neuro-Fuzzy and Eigenface. International Journal of Computer Science and Engineering (IJCSE), 5(4), 1-10.

19. Jonathan Huang, Vivek Rathod, Chen Sun, MenglongZhu, AnoopKorattikara, AlirezaFathi, Ian Fischer,ZbigniewWojna, Song Yang, Sergio Guadarrama, et al.,Speed/accuracy trade-offs for modern convolutionalobjectdetectors,IEEECVPR,4,,2017.

20. www.electronicscomp.com

21. KALAISELVI, B., \& ANANDAMALA, G. INTELLIGENT FACIAL RECOGNITION AND RETRIEVAL FROM FACIAL IMAGE DATABASES.

22. Zhong-QiuZhao,PengZheng,ShoutaoXu,Wu.Xindong,Objectdetectionwithdeeplearning:Areview,IEEETransactionsonNeuralNetworks andLearningSystems, 2019. 